\title{
Introduction to the mini-review series on mitochondrial matters in epilepsy
}

\author{
George H. Sack Jr.
}

Published online: 14 December 2010

(C) Springer Science+Business Media, LLC 2010

\begin{abstract}
Dysfunction of neuronal metabolism is central to all forms of seizure activity. Clinical, genetic and metabolic studies have implicated mitochondria in the process. Alterations in electron transport, generation of reactive oxygen species, calcium metabolism and apoptosis all have been described in human and animal nervous tissue and reflect changes in mitochondrial physiology. Improved understanding of the molecular details underlying seizures has begun to provide rational approaches to the design of new treatment strategies.
\end{abstract}

Keywords Seizure $\cdot$ Mitochondrion $\cdot$ Reactive oxygen species $\cdot$ Mutation $\cdot$ Apoptosis $\cdot$ Electron transport

The dramatic clinical presentation of many seizure disorders has been recognized for millennia. It was described by the Greeks who developed the term epilepsy (meaning "seized upon") which is still used. Old reports also refer to the "falling sickness." Over the years, the term "epilepsy" has acquired negative connotations, and "seizure" is now preferred. Many prominent individuals have been affected including Julius Caesar, James Madison, Peter Tchaikovsky, Alfred Nobel and Leo Tolstoy.

Seizures vary with respect to neurologic involvement and severity, ranging from minimal changes or "absence spells" to florid, generalized muscle jerking and loss of consciousness. The latter can occasionally be life-threatening. Because of the prominence of seizures (estimates of affected individuals in

\footnotetext{
G. H. Sack Jr. $(\bowtie)$
}

Departments of Medicine and Biological Chemistry,

The Johns Hopkins University School of Medicine,

725 N. Wolfe Street, Physiology 615,

Baltimore, MD 21205, USA

e-mail: gsack@jhmi.edu the population are as high as $1 \%$ ) and the long history of observing affected individuals a considerable body of detailed clinical descriptions is available. Distinguishing types of seizures has helped direct treatment, clarify prognosis and, more recently, provide insight into basic pathophysiology.

Clearly, the generic "seizure" involves neuronal dysfunction. Often, there are no physical changes in brain cells. Alternatively, evidence of earlier brain injury, gliosis, vascular changes and scarring in brain tissue may suggest a large-scale structural problem. Nutritional and toxic aspects of seizures have been studied in past decades. Perhaps more valuable in terms of cellular biology have been insights gained from examination of well-defined seizure types.

Of particular interest has been a group of neuromuscular disorders genetically linked to the mitochondrial genome. These are reviewed by Zaurka and Kunz (2010) and have several important features including predictable familial transmission patterns (via maternal germ line), defined mutation site(s) as well as frequent derangements of electron transport, membrane potential and calcium signaling. Their study has been complicated by the multiplicity of copies of mitochondrial chromosomes in each cell. In conditions of heteroplasmy (where only some of the mitochondrial genomes contain mutation[s]) the onset and severity of dysfunction may be difficult to predict. Often, it appears that there may be thresholds of intracellular compensation, beyond which cell failure occurs.

Other, apparently inherited, forms of abnormal mitochondrial activity underlie a group of disorders with early onset, currently categorized as early infantile epileptic encephalopathy (EIEE). Affected individuals have poor treatment responses and generally psychomotor retardation. Molinari (2010) presents details of individuals with EIEE from several kindreds. In one category are those with defective complex I electron transport function; another 
type has abnormal complex IV function. By contrast, others have defined mutations in a mitochondrial glutamate transporter SLC25A22.

In addition to inherited forms of mitochondrial-based seizure disorders, there is evidence that reactive oxygen species (ROS) generated within the electron transport chain can, by themselves, lead to protein/lipid/carbohydrate modifications. In addition, ROS can lead to mutations (frequently deletions) of the circular mitochondrial DNA with subsequent loss or modification of function(s). Details of some of the reaction products and the affected pathways are noted by Waldbaum and Patel (2010) and Chang and Yu (2010). As noted for the inherited forms of such conditions, heteroplasmy likely complicates the development of the observable clinical endpoint of overt seizures. In fact, more subtle neuronal metabolic change(s) likely antedate gross, transmissible seizure activity. Muscle dysfunction (and structural changes) as well as cognitive derangement may develop as well. Precise measurements of small, presumably local, nerve cell changes have been difficult to make but approaches such as scanning for fluoro-deoxyglucose metabolism are promising.
Another important cellular pathway that can result from mitochondrial change(s) is apoptosis. Cell death can lead to loss of information transmission as well as regional damage associated with release of metabolites (either native or modified) as well as ROS. Cell death has been observed in local brain regions (Chen et al. 2010) and the central role(s) of mitochondria in this process is well-established.

Applying detailed molecular analysis already has incriminated mitochondrial structure and function in the pathophysiology of seizure activity and has suggested possible therapeutic approaches. Further study is likely to elucidate more details of this age-old affliction.

\section{References}

Chang SJ, Yu BC (2010) J Bioenerg Biomem

Chen S-D, Chang AYW, Chuang Y-C (2010) J Bioenerg Biomem Molinari F (2010) J Bioenerg Biomem

Waldbaum S, Patel A (2010) J Bioenerg Biomem

Zaurka G, Kunz WS (2010) J Bioenerg Biomem 